\title{
Wafer-Scale Ion Beam Lithography of Nanopore Devices
}

\author{
J. Klingfus ${ }^{1}$, A. Nadzeyka ${ }^{2}$, S. Bauerdick ${ }^{2}$, T. Albrecht ${ }^{3}$ and J. B. Edel ${ }^{3}$ \\ ${ }^{1 .}$ Raith USA, Inc. Ronkonkoma, New York 11779 USA \\ 2. Raith GmbH, 44263 Dortmund Germany \\ 3. Department of Chemistry, Imperial College London, London SW7 2AZ UK
}

The quality and repeatability for fabrication of membrane-based solid state nanopore devices for applications such as DNA sequencing, molecule analysis and biological filtering is of scientific as well as commercial interest [1]. Pores used for single molecule transit can be judged by many attributes; they should be $<20 \mathrm{~nm}$, of high quality (high degree of roundness), and have high aspect ratio. If pores are fabricated by direct ion milling, care should be taken to minimize substrate damage and contaminant ion implantation. Maintaining tight control over distribution of size and quality is also important for device response repeatability and therefore places certain constraints on the fabrication process as well as the instrumentation used during the fabrication (namely various stability aspects of the instrument.)

Solid state membrane-based nanopore devices have been fabricated using various direct write approaches (ion mill based like FIB-SEM and HIM, but also TEM) and resulted in varying degrees of quality, precision, repeatability and yield [1]. Here we report on nanopore results obtained using the Raith ionLiNE instrument, a FIB tool designed exclusively and foremost as a lithography nanofabrication instrument.

The ionLiNE tool is designed with a highly accurate laser interferometer sample stage, and a column designed to run very stable over long periods of time. Merits of the high beam fidelity, stability, and unique beam movement (patterning) strategies have been reported here previously [2]. The truly lithography-centric software architecture of the ionLiNE results in design file and work-flow techniques which are cross-compatible and complimentary to electron beam lithography (EBL) and optical lithography instrumentation. Focused ion beam lithography (IBL) thus gains greater attention as a viable and more-widely used nanofabrication processing avenue.

Nanopore devices are fabricated in an automated way across a 4 inch wafer containing silicon nitride membranes (Figure 1.) In a step-and-repeat manner, nanopores are milled precisely within each prefabricated device, the goal of which is to place the nanopore directly between a set of readout electrodes which were created using previous fabrication steps (Figure 2.) The laser interferometer accuracy ensures correct pore placement. An automated height sensing system is used to maintain consistent beam focus while traveling over the large lateral dimension of the wafer. Results are given of high quality and repeatable $20 \mathrm{~nm}$ pores on $100 \mathrm{~nm}$ thick Si3N4 membranes which are distributed across the entirety of a 4 inch wafer. 


\section{References:}

[1] J. Edel, T. Albrecht, Nanopores for Bioanalytical Applications: Proceedings of the First International Conference (ROYAL SOC OF CHEMISTRY, 2012).

[2] J. Sanabia et al, Focused Ion Beam Lithography of Nanophotonic Structures: Micoscopy and Microanalysis 18 (2012), p. 612.

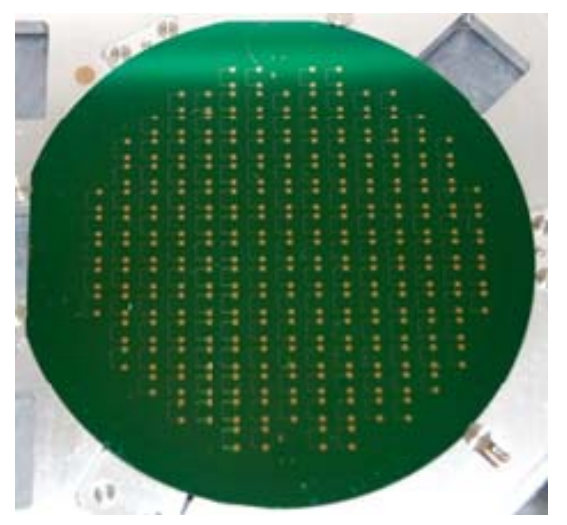

Figure 1. A 4 inch wafer of 175 devices. Visible are connection pads and read-out electrodes

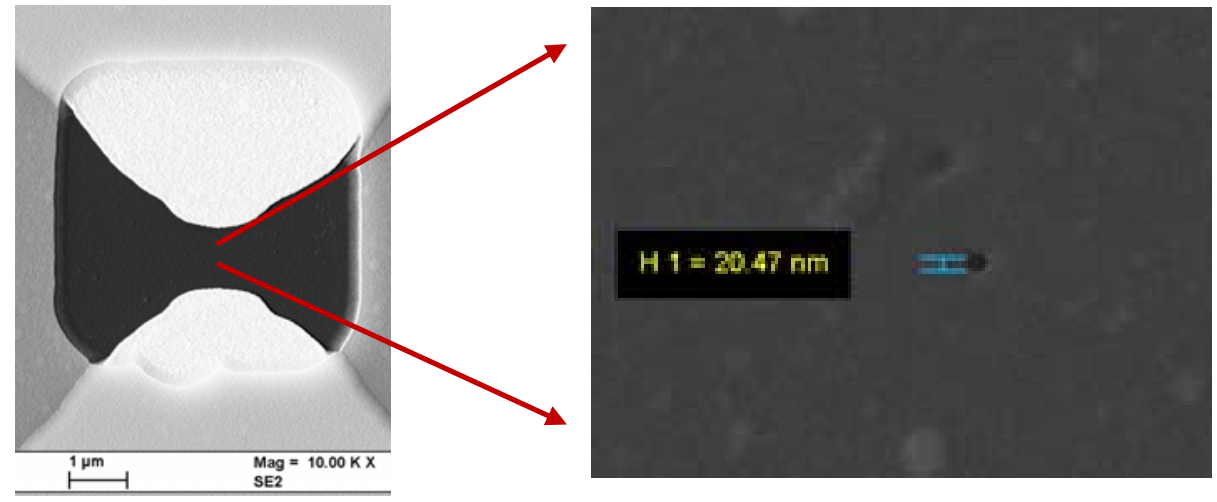

Figure 2. $20 \mathrm{~nm}$ pore in $100 \mathrm{~nm}$ thick Si3N4 membrane between read-out electrodes of single device.

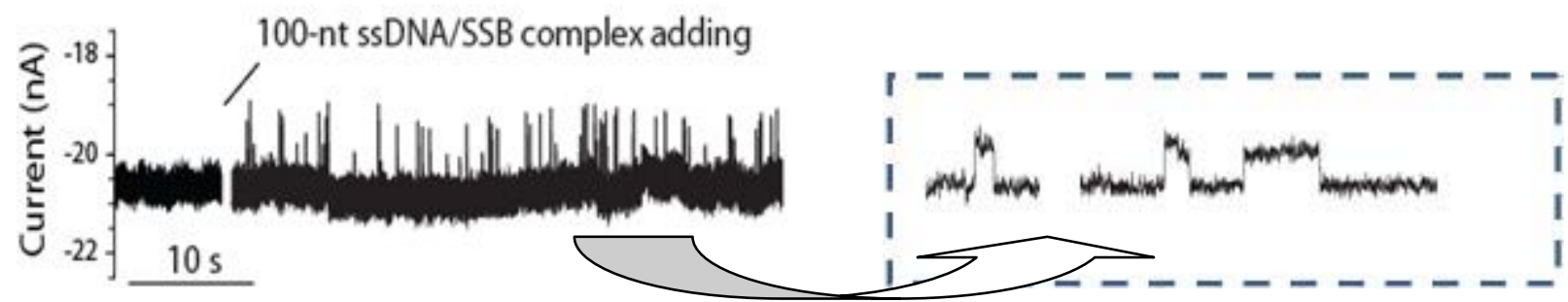

Figure 3. Example of nanopore measurement of 100 nucleotide single strand DNA. 